UM-P-96/74

RCHEP-96/07

Revised Version

\title{
Higher Twist Effect in Inclusive Quarkonium Photoproduction
}

\author{
J.P. Ma \\ Recearch Center for High Energy Physics \\ School of Physics \\ University of Melbourne \\ Parkville, Victoria 3052 \\ Australia \\ E-Mail: ma@physics.unimelb.edu.au
}

\begin{abstract}
:
We analyze higher twist effect in photoproduction of quarkonium, where the quarkonium is a spin-triplet, S-wave state. We find that the nonperturbative effect of next-toleading twist is contained in three correlation functions related to the initial hadron and some of the effect of next-to-leading twist is only suppressed by the inverse of the mass square of the quarkonium. A naive estimation indicates that the effect can be significant for charmonium. In the analysis we only take the leading order in the small velocity expansion for the nonperturbative parts related to the quarkonium. Possible corrections from higher orders, especially, corrections from color-octet states are discussed in detail.
\end{abstract}

PACS Numbers: 12.38.Bx, 13.85.Ni, 14.40.Gx.

Keywords: Twist-4 effect, quarkonium production. 


\section{Introduction}

Recently the theory of quarkonium production has been developed rapidly(See recent reviews given in [1]). Quarkonium production can be regarded as a two-step process, where a heavy quark $Q$ and its antiquark $\bar{Q}$ are produced and then this $Q \bar{Q}$ pair is transmitted into a quarkonium. The production of the $Q \bar{Q}$ pair can be treated with perturbative QCD because the mass $M_{Q}$ of $Q$ is large, while the transition is a nonperturbative process. It is realized that in a rest frame of the quarkonium the quark $Q$ or the antiquark $\bar{Q}$ moves with a small velocity $v$. Hence a small velocity expansion can be used to treat the nonperturbative transition. As a result the effect of the transition can be described by quarkonium matrix elements of local operators defined in nonrelativistic QCD[2]. It is interesting to note that not only a color-singlet $Q \bar{Q}$, but also a color-octet $Q \bar{Q}$ can be transmitted into a quarkonium. This is in contrast to the color-singlet model and is specially of importance to $\mathrm{P}$-wave quarkonium production, because the production rates through a color-singlet and through a color-octet are at the same order of $v$. For $S$-wave quarkonium, although the contributions from a color-octet $Q \bar{Q}$ is suppressed at least by $v^{2}$ in comparison with those from a color-singlet, they can be significant[1]. Besides this nonperturbative effect related to the transition there is another nonperturbative effect if there are hadrons in the initial state. This nonperturbative effect can be analyzed with a twist expansion. At the order of leading twist it results in the well-known parton model. The subject of the present work is to analyze the effect from the order of next-to-leading twist in inclusive photoproduction of quarkonium.

In the case of inclusive photoproduction of a single jet with a large transversal momentum $P_{T}$, one can expect that higher twist effects are suppressed by the power of $P_{T}^{-1}$, since $P_{T}$ is the only large scale in the problem. If one observes instead of a single jet a quarkonium state, there is, in addition to $P_{T}$, another large scale-the mass of the heavy quark $M_{Q}$. Naturely one expects that some effects from higher twist may be only suppressed by the power of $M_{Q}^{-1}$. In this work we assume that no polarization is observed, so the powers will be even numbers. We find that some effect of next-to-leading twist is suppressed indeed by $M_{Q}^{-2}$. This indicates that the effect from higher twist may be substantial in the production of charmonium, as the mass $M_{c}$ is not so large.

In this work we only consider the quarkonium as a S-wave, spin-triplet state and denote it as $\psi$. For charmonium and bottonium it corresponds to $J / \psi$ and to $\Upsilon$ respectively. We will assume that the nonperturbative parts related to the quarkonium and to the initial hadron can be factorized separately and analyze in detail the effect of next-to-leading twist 
in the $\psi$-production, where the formation of $\psi$ from a $Q \bar{Q}$ pair is considered at leading order of $v^{2}$. At this order, only a color-singlet $Q \bar{Q}$ can be transmitted into $\psi$. At higher orders of $v$ there are relativistic corrections and contributions from color-octet states of $Q \bar{Q}$ pair. We will discuss this in length in a separate section, where we also discuss electroproduction briefly. To analyze the nonperturbative effect related to the initial hadron we employ the diagram expansion method. This method was first used to classify higher twist effects in deeply inelastic scattering[3], it exactly delivers the same results as those obtained with traditional Wilson operator expansion[4]. This method was also applied or extended to other processes. An incompleted list of references can be found in [5-10]. However, the extension of the method to our case is not quite straightforward. In deeply inelastic scattering, because the process is totally inclusive, the perturbative parts of diagrams with different numbers of incoming glue as partons can be related to each other with the help of low energy theorems[3], basically derived from the Ward identity. This leads to that the contributions from diagrams with a given number $N$ of incoming glue and from diagrams with $N+1$ incoming glue can be combined together by replacing a space-time derivative in the corresponding nonperturbative part with a gauge covariant derivative. Hence $S U(3)$ gauge symmetry is maintained explicitly. The process we consider is seminclusive, where a $Q \bar{Q}$ pair is observed indirectly, thus the corresponding low-energy theorems are lacking. Further, the perturbative parts are much more complicated than those in deeply inelastic scattering. In this work we will only consider in the diagram expansion those diagrams whose perturbative parts are at the same order of coupling constants and will simply replace space-time derivatives in nonperturbative parts by gauge covariant derivatives.

In our work we will assume that we do not observe any polarization of any particle, and we take only leading order in coupling constants. We will also assume that the produced $\psi$ has moderate transverse momentum, i.e., the $\psi$ is not in the forward region in a fixed target experiment. In this region various processes can produce $\psi$, some of them can not be described with perturbative QCD. We neglect heavy flavor content in the initial hadron, which is expected to give small contribution. An interesting aspect of the processes is that at this order only glue are responsible for the quarkonium production. Studying these processes will help to understand the role played by gluon in hadrons. The process considered here was used to study gluon distribution in nucleon before(See [11] and references cited there).

Our work is organized as the following: In Sect.2 we introduce notations and the diagrams in the diagram expansion which are needed to be considered. The results at the leading twist is also given. In Sect.3 we work out the results for next-to-leading twist. A discussion of our results is presented. In Sect.4 we will discuss possible contributions from 
higher orders of $v$, where production of a color-octet $Q \bar{Q}$ pair can also lead to contributions for the total production rate. In this section electroproduction is also discussed briefly. We summarize our work in Sect.5.

\section{The Diagram Expansion and Results of Leading Twist}

We consider the inclusive process:

$$
\gamma(q)+H(P) \rightarrow \psi(k)+X
$$

The momenta of particles are given in the brackets. We choose a frame in which the hadron $H$ moves in the $z$-direction and the photon in the opposite direction. The photon is on-shell and $H$ has mass $M_{H}$. Throughout our work we will neglect $M_{H}$. The correction introduced by the target mass may be included by using Nachtmann variable[12]. In our approximation the quarkonium mass $M_{\psi}$ is twice of $M_{Q}$. We define $s=(q+P)^{2}$ and $t=(P-k)^{2}$. For performing the analysis it is convenient to use light-cone coordinate system. In this system a 4 -vector is expressed as $p^{\mu}=\left(p^{+}, p^{-}, p^{1}, p^{2}\right)=\left(p^{+}, p^{-}, \mathbf{p}_{\mathbf{T}}\right)$. The relation to conventional expression is $p^{+}=\frac{1}{\sqrt{2}}\left(p^{0}+p^{3}\right), p^{-}=\frac{1}{\sqrt{2}}\left(p^{0}-p^{3}\right)$. In the system the photon carries the momentum $q^{\mu}=\left(0, q^{-}, 0,0\right)$ and $H P^{\mu}=\left(P^{+}, \frac{M_{H}^{2}}{2 P^{+}}, 0,0\right)$. We introduce in the frame two vectors $n$ and $\ell$ and a tensor $d_{T}^{\mu \nu}$ :

$$
\begin{aligned}
n^{\mu} & =(0,1,0,0), \quad \ell^{\mu}=(1,0,0,0), \\
d_{T}^{\mu \nu} & =g^{\mu \nu}-n^{\mu} \ell^{\nu}-n^{\nu} \ell^{\mu} .
\end{aligned}
$$

Contracting any vector with $d_{T}^{\mu \nu}$ gives its transverse part. We will work in the light-cone gauge $n \cdot G(x)=G^{+}(x)=0$, where $G^{\mu}(x)=G^{a, \mu}(x) T^{a}$ is the gluon field. In this gauge a component of the gluon field strength tensor $G^{\mu \nu}$ takes a simple form:

$$
G^{a,+\mu}(x)=\frac{\partial}{\partial x^{-}} G^{a, \mu}(x) .
$$

To analyze the effect upto next-to-leading twist we need to consider the diagrams given in Fig.1A, Fig.1B and Fig.1C in the diagram expansion approach. In these diagrams the black boxes represents the nonperturbative part involved by the hadron $H$, the upper parts, i.e., the blank boxes, contain the perturbative part for production of the $Q \bar{Q}$ pair at the leading order of coupling constants and the transition of the pair into $\psi$, they represent the sample of Feynman diagrams given in Fig.2A, Fig.2B and Fig.2C correspondingly. These diagrams are with Cutkosky cut since we are interested in cross section. With 
twist-classification, Fig.1A has the leading twist 2, Fig.1B has 3 and Fig.1C has 4. The contribution from Fig.1A can be written as:

$$
\begin{aligned}
& 2 s d \sigma_{A}= \frac{d^{4} k}{(2 \pi)^{4}} 2 \pi \delta\left(k^{2}-M_{\psi}^{2}\right) \int \frac{d^{4} p_{1}}{(2 \pi)^{4}} A_{\mu \nu}^{a b}\left(p_{1}\right) \\
& \cdot \int d^{4} x e^{-i x \cdot p_{1}}<H(P)\left|G^{a, \nu}(x) G^{b, \mu}(0)\right| H(P)>
\end{aligned}
$$

In Eq.(2.4) $A_{\mu \nu}^{a b}$ corresponds to the upper part in Fig.1A, this part is the contribution from diagrams given in Fig.2A. The hadronic matrix element in Eq.(2.4) corresponds to the black box in Fig.1A and it is averaged over the spin of $H$. Because of the color symmetry we can write Eq.(2.4) as

$$
\begin{aligned}
2 s d \sigma_{A} & =\frac{d^{4} k}{(2 \pi)^{4}} 2 \pi \delta\left(k^{2}-M_{\psi}^{2}\right) \frac{1}{8} \int \frac{d^{4} p_{1}}{(2 \pi)^{4}} S_{\mu \nu}\left(p_{1}\right) \\
\cdot \int d^{4} x e^{-i x \cdot p_{1}}<H(P)\left|G^{b, \nu}(x) G^{b, \mu}(0)\right| H(P)> & \\
S_{\mu \nu}\left(p_{1}\right) & =A_{\mu \nu}^{a a}\left(p_{1}\right)
\end{aligned}
$$

The contribution from Fig.1C takes the form:

$$
\begin{aligned}
& 2 s d \sigma_{C}= \frac{d^{4} k}{(2 \pi)^{2}} 2 \pi \delta\left(k^{2}-M_{\psi}^{2}\right) \int \frac{d^{4} k_{1}}{(2 \pi)^{4}} \frac{d^{4} k_{2}}{(2 \pi)^{4}} \frac{d^{4} k_{3}}{(2 \pi)^{4}} C_{\mu_{1} \mu_{2} \mu_{3} \mu_{4}}^{a_{1} a_{2} a_{3} a_{4}}\left(k_{1}, k_{2}, k_{3}\right) \\
& \cdot \int d^{4} x_{1} d^{4} x_{2} d^{4} x_{3} e^{-i x_{1} \cdot k_{1}-i x_{2} \cdot x_{2}+i x_{3} \cdot k_{3}} \\
& \quad<H(P)\left|G^{a_{1}, \mu_{1}}\left(x_{1}\right) G^{a_{2}, \mu_{2}}\left(x_{2}\right) G^{a_{3}, \mu_{3}}\left(x_{3}\right) G^{a_{4}, \mu_{4}}(0)\right| H(P)>
\end{aligned}
$$

where $C$ is the upper part and corresponds to the diagrams given in Fig.2C. Note $C$ and $A$ are at the same order of $\alpha$ and $\alpha_{s}$. At the leading twist the momenta $k_{i}^{\prime} \mathrm{s}$ in the perturbative part $C$ will be set to be proportional to $P$, so the quarkonium will be produced with zero transversal momentum. We will not consider this type of contribution as mentioned in the introduction. As for the contribution from Fig.1B, the perturbative part is one order higher in $g_{s}$ than those in Fig.1A. Again we will not consider it. It is worth to point out that some contributions from diagrams at higher order of $g_{s}$ will be included in our final results, because we later replace space-time derivatives with gauge covariant derivatives for gauge invariance. In the case we consider the contribution to the process is only from Fig.1A with the perturbative part specified with the Feynman diagrams given in Fig.2A. The main task for analyzing twist- 4 effect is to separate them from the contribution from Fig.1A.

To achieve the separation one observes that the space-time dependence of the matrix elements in Eq.(2.5) is controlled by different scales in different directions. In --direction 
this scale is $P^{+}$and it is very large. In +-direction and transverse direction this scale is just $P^{-}$or $\Lambda_{Q C D}$, the $\Lambda$ - parameter of QCD, which are small. With this observation one can approximate the $x^{+}$- and $\mathbf{x}_{\mathbf{T}}$-dependence by Taylor-expanding $x^{+}$and $\mathbf{x}_{\mathbf{T}}$. This expansion is equivalent to the expansion of $S_{\mu \nu}\left(p_{1}\right)$ around $p_{1}^{\mu}=\hat{p}_{1}^{\mu}=\left(p_{1}^{+}, 0,0,0\right)$. The expansion reads:

$$
\begin{aligned}
S_{\mu \nu}\left(p_{1}\right)= & S_{\mu \nu}\left(\hat{p}_{1}\right)+\left.\frac{\partial S_{\mu \nu}\left(p_{1}\right)}{\partial p_{1}^{\alpha}}\right|_{p_{1}=\hat{p}_{1}} \Delta p_{1}^{\alpha} \\
& +\left.\frac{1}{2} \frac{\partial^{2} S_{\mu \nu}\left(p_{1}\right)}{\partial p_{1}^{\alpha} \partial p_{1}^{\beta}}\right|_{p_{1}=\hat{p}_{1}} \Delta p_{1}^{\alpha} \Delta p_{1}^{\beta}+\cdots
\end{aligned}
$$

where $\Delta p_{1}=p_{1}-\hat{p}_{1}$. The $\cdots$ denotes higher order in $\Delta p_{1}$ which will lead contributions with twist higher than 4 . The expansion is called as collinear expansion. The leading twist contribution comes only from the first term in the expansion. This term does not depend on $p_{1}^{-}$and $\mathbf{p}_{\mathbf{1} \mathbf{T}}$. Taking this term for $S_{\mu \nu}$ in Eq.(2.5) we can perform the integration over $p_{1}^{-}$and $\mathbf{p}_{\mathbf{1} \mathbf{T}}$ directly:

$$
\begin{aligned}
2 s d \sigma_{A} & =\frac{d^{4} k}{(2 \pi)^{4}} 2 \pi \delta\left(k^{2}-M_{\psi}^{2}\right) \frac{1}{8} \int \frac{d z}{z} S_{\mu \nu}\left(\hat{p}_{1}\right) \\
& \cdot \frac{z P^{+}}{2 \pi} \int d x^{-} e^{-i z P^{+} x^{-}}<H(P)\left|G^{b, \nu}\left(x^{-} n\right) G^{b, \mu}(0)\right| H(P)> \\
& + \text { "Higher Twist Terms" }
\end{aligned}
$$

where we made the substitution $p_{1}^{+}=z P^{+}$. Because of the symmetries of Lorentz boost along $z$-direction and of rotation around $z$-axis, the Fourier transformed matrix element can be written:

$$
\begin{aligned}
\frac{z P^{+}}{\pi} \int d x^{-} e^{-i z P^{+} x-} & <H(P)\left|G^{b, \nu}\left(x^{-} n\right) G^{b, \mu}(0)\right| H(P)>= \\
& d_{T}^{\mu \nu} \frac{z P^{+}}{2 \pi} \int d x^{-} e^{-i z P^{+} x^{-}}<H(P)\left|G^{b, \rho}\left(x^{-} n\right) G^{b}{ }_{\rho}(0)\right| H(P)> \\
& +n^{\mu} n^{\nu} \frac{z P^{+}}{\pi} \int d x^{-} e^{-i z P^{+} x^{-}}<H(P)\left|G^{b,-}\left(x^{-} n\right) G^{b,-}(0)\right| H(P)>
\end{aligned}
$$

where $d_{T}^{\mu \nu}$ is given in Eq.(2.2). In light-cone gauge the dynamically independent gluon fields are $G^{1}$ and $G^{2}, G^{-}$can be solved with the equation of motion and the term in Eq.(2.9) with $G^{-}$will be shown in the next section to be at higher twist. The leading twist contribution from Fig.1A can be written now as:

$$
\begin{aligned}
2 s d \sigma_{A} & =\frac{d^{4} k}{(2 \pi)^{4}} 2 \pi \delta\left(k^{2}-M_{\psi}^{2}\right) \int \frac{d z}{z} f_{G / H}(z) \cdot\left[-\frac{1}{16} d_{T}^{\mu \nu} \cdot S_{\mu \nu}\left(\hat{p}_{1}\right)\right] \\
& +" \text { Higher Twist Terms" }
\end{aligned}
$$


The term in $[\ldots]$ is just the probability for the photon-gluon fusion into $\psi$ and a gluon, where the initial gluon is on shell because of $\hat{p}_{1}^{2}=0$. Therefore the interpretation of parton model applies. The function $f_{G / H}(z)$ can be read from Eq.(2.9):

$$
f_{G / H}(z)=-\frac{z P^{+}}{2 \pi} \int d x^{-} e^{-i z P^{+} x^{-}}<H(P)\left|G^{b, \rho}\left(x^{-} n\right) G_{\rho}^{b}(0)\right| H(P)>.
$$

Because of the total momentum conservation in + direction the variable $z$ is constrained between 0 and 1 . The function $f_{G / H}(z)$ is positive and can be interpreted as the probability of finding a gluon in $H$ with the energy fraction $z$. It is preferred to use strength tensor instead gluon field to define $f_{G / H}(z)$. Using Eq.(2.3) one can obtain:

$$
f_{G / H}(z)=\frac{-1}{2 \pi z P^{+}} \int d x^{-} e^{-i z P^{+} x^{-}}<H(P)\left|G^{b,+\rho}\left(x^{-} n\right) G_{\rho}^{b,+}(0)\right| H(P)>
$$

This is the definition of gluon distribution given in [13] in light-cone gauge. For arbitrary gauge one needs to supply Wilson line operator between the two tensor operators.

The perturbative result at the leading twist is well known. For completeness we will give this result. For this purpose we introduce

$$
\begin{aligned}
\hat{s} & =\left(q+\hat{p}_{1}\right)^{2}, \quad \hat{t}=\left(k-\hat{p}_{1}\right)^{2}, \\
\sigma & =\sigma^{(0)}+\sigma^{(2)}+\cdots .
\end{aligned}
$$

$\sigma^{(0)}$ denotes the contribution at leading twist. $\sigma^{(2)}$ stands for the contribution at twist4. The invariant $\hat{s}$ and $\hat{t}$ by neglecting $M_{H}$ are simply related to $s$ and $t$ via $\hat{s}=z s$ and $\left(\hat{t}-M_{\psi}^{2}\right)=z\left(t-M_{\psi}^{2}\right)$ respectively. The result at leading twist is:

$$
\begin{aligned}
\frac{d \sigma^{(0)}}{d t}= & \frac{32 \pi}{3} \alpha \alpha_{s}^{2} Q_{Q}^{2} M_{\psi}\left|R_{\psi}(0)\right|^{2} \int \frac{d z}{z} f_{G / H}(z) \\
& \cdot \frac{\hat{s}^{2}\left(\hat{s}-M_{\psi}^{2}\right)^{2}+\hat{t}^{2}\left(\hat{t}-M_{\psi}^{2}\right)^{2}+(\hat{s}+\hat{t})^{2}\left(\hat{s}+\hat{t}-M_{\psi}^{2}\right)^{2}}{\hat{s}^{2}\left(\hat{s}-M_{\psi}^{2}\right)^{2}\left(\hat{t}-M_{\psi}^{2}\right)^{2}(\hat{s}+\hat{t})^{2}}
\end{aligned}
$$

where $Q_{Q}$ is the electric charge of $Q$ in unit $e . R_{\psi}(0)$ is the radial wavefunction for $\psi$ at the origine, which is related to a matrix element defined in [2].

The contribution from the next-to-leading twist is from the term with $n^{\mu} n^{\nu}$ in Eq.(2.9) and the terms with derivatives in Eq.(2.7). We will analyze them in the next section. 


\section{The Results at the Next-to-leading Twist}

We start first with the term with $n^{\mu} n^{\nu}$ in Eq.(2.9). This term corresponds to the contribution from the nonpropagating part of the gluon propagator[5]. In this term only the --components of gluon fields are involved and they are not dynamically independent. With the equation of motion in QCD for these component one can solve them in terms of transverse components of gluon fields and quark fields. The equation of motion reads:

$$
\frac{\partial G^{a,+-}(x)}{\partial x^{-}}=-\left(D_{T}^{\mu} G_{\mu}^{+}(x)\right)^{a}-g_{s} J^{a,+}(x),
$$

where $D_{T}^{\mu}=d_{T}^{\mu \nu} D_{\nu}$ and $D_{\nu}$ is the covariant derivative in the adjoin representation. $J^{a,+}$ is the +-component of quark color current. Assuming all fields approach zero at infinite space-time, one can solve this equation formally:

$$
G^{a,+-}\left(x^{+}, x^{-}, \mathbf{x}_{\mathbf{T}}\right)=-\frac{1}{2} \int_{-\infty}^{+\infty} d \xi \tau\left(x^{-}-\xi\right)\left\{\left(D_{T}^{\mu} G_{\mu}^{+}\left(x^{+}, \xi, \mathbf{x}_{\mathbf{T}}\right)\right)^{a}+g_{s} J^{a,+}\left(x^{+}, \xi, \mathbf{x}_{\mathbf{T}}\right)\right\} .
$$

The function $\tau(z)$ equals 1 for $z>0$ and -1 for $z \leq 0$. To use this solution we write $G^{-}$into $G^{+-}$in the second term in Eq.(2.9) with help of Eq.(2.3) by partial integration. Finally we obtain the contribution from this term:

$$
2 s d \sigma_{A 1}=\frac{d^{4} k}{(2 \pi)^{4}}(2 \pi) \delta\left(k^{2}-M_{\psi}^{2}\right) \int \frac{d z}{z} e_{1}(z)\left\{\frac{1}{8} \frac{n^{\mu} n^{\nu}}{\left(n \cdot \hat{p}_{1}\right)^{2}} S_{\mu \nu}\left(\hat{p}_{1}\right)\right\}
$$

where the function $e_{1}(z)$ is defined as

$$
\begin{array}{r}
e_{1}(z)=\frac{1}{2 \pi z P^{+}} \int d x^{-} e^{-i z P^{+} x^{-}}<H(P) \mid\left[\left(D_{T}^{\mu} G_{\mu}^{+}\left(x^{-} n\right)\right)^{a}+g_{s} J^{a,+}\left(x^{-} n\right)\right] \\
\cdot\left[\left(D_{T}^{\nu} G_{\nu}^{+}(0)\right)^{a}+g_{s} J^{a,+}(0)\right] \mid H(P)>.
\end{array}
$$

This function is positive and has support only for $z$ between 0 and 1 . It has a dimension 2 in mass while $f_{G / H}(z)$ is dimensionless. Therefore this term will lead to contribution at order of next-to-leading twist. We used subscript $A 1$ in Eq.(3.3) to denote this contribution. The perturbative part in this contribution is the part in $\{\cdots\}$ in Eq.(3.3) and can be calculated straightforward. We obtain:

$$
\begin{aligned}
\frac{d \sigma_{A 1}}{d t} & =\frac{32 \pi}{3} \alpha \alpha_{s}^{2} Q_{Q}^{2} M_{\psi}\left|R_{\psi}(0)\right|^{2} \int \frac{d z}{z} \cdot \frac{e_{1}(z)}{M_{\psi}^{2}} \cdot \frac{1}{\hat{s}^{3}\left(\hat{s}-M_{\psi}^{2}\right)^{2}\left(\hat{t}-M_{\psi}^{2}\right)^{2}(\hat{s}+\hat{t})^{2}} \\
& \cdot\left\{2 \hat{s}^{3}(\hat{s}+\hat{t})^{2}-4 M_{\psi}^{2}\left(\hat{s}^{4}+\hat{s}^{3} \hat{t}+2 \hat{s} \hat{t}^{3}+2 \hat{t}^{4}\right)+2 M_{\psi}^{4}\left(\hat{s}^{3}+\hat{s} \hat{t}^{2}+4 \hat{t}^{3}\right)\right\}
\end{aligned}
$$

Comparing this contribution with that from the leading twist in Eq.(2.14) there is an extra $M_{\psi}^{-2}$ term, the contribution hence may be suppressed by this power. 
Next we consider the contribution from the terms with derivatives in the collinear expansion in Eq.(2.7). The second term in Eq.(2.7) leads a contribution which can be written as:

$$
\begin{aligned}
& \frac{d^{4} k}{(2 \pi)^{4}}(2 \pi) \delta\left(k^{2}-M_{\psi}^{2}\right) \int \frac{d^{4} p_{1}}{(2 \pi)^{4}} \frac{1}{16}\left[\left.\frac{\partial S_{\mu \nu}}{\partial p_{1}^{\alpha}}\right|_{p_{1}=\hat{p}_{1}}\right] \\
& \cdot \quad(-i) \int d^{4} x e^{-i x \cdot p_{1}}<H(P)\left|\left(\partial^{\alpha} G^{b, v}(x)\right) G^{b, \mu}(0)+G^{b, \nu}(0)\left(\partial^{\alpha} G^{b, \mu}(-x)\right)\right| H(P)>,
\end{aligned}
$$

where we have used partial integration and translational invariance for the hadronic matrix element. Since we do not observe any spin of any particle, by using P(Parity)- and T(Time reversal)- symmetries one can show that:

$$
S_{\mu \nu}\left(p_{1}\right)=S^{\nu \mu}\left(p_{1}\right)
$$

while the matrix element is antisymmetric in $\mu \nu$ in contrast to Eq.(3.7). Hence the second term in Eq.(2.7) does not contribute. If any polarization is observed, this term will lead to a twist-3 effect.

The third term in Eq.(2.7) leads to a contribution given by:

$$
\begin{aligned}
\int \frac{d^{4} k}{(2 \pi)^{4}}(2 \pi) \delta\left(k^{2}-M_{\psi}^{2}\right) \int \frac{d p_{1}^{+}}{2 \pi} \frac{1}{16}\left[\left.\frac{\partial^{2} S_{\mu \nu}\left(p_{1}\right)}{\partial p_{1}^{\alpha} \partial p_{1}^{\beta}}\right|_{p_{1}=\hat{p}_{1}}\right] \\
\cdot(-1) \int d x^{-} e^{-i x^{-} p_{1}^{+}}<H(P)\left|G^{b, \nu}(x)\left(\partial^{\alpha} \partial^{\beta} G^{\mu}(0)\right)^{b}\right| H(P)>.
\end{aligned}
$$

It should be noted that by using the translational invariance one can let the derivatives acting on the gluon fields differently than that given above and hence there is an ambiguity to form operators in the matrix element. We take the above form since the operator is just the trace term subtracted in the corresponding twist-2 operator. The leading contribution in Eq.(3.7) comes from those terms in which the contracting Lorentz indices $\mu, \nu, \alpha$ and $\beta$ are only the transverse indices. If any of them is -, one can show that the contribution is at higher twist. For $\mu$ or $\nu$ being - the argument is the same as for the second term in Eq.(2.8). For $\alpha$ or $\beta$ in the matrix element being - one can show because of the covariance of Lorentz boost along $z$-direction that the corresponding contribution is suppressed by the factor $\frac{1}{P^{+}}$relative to the terms with $\alpha$ or $\beta$ being transverse indices. Similarly as to Eq. (2.9) one can write the Fourier transformed matrix element in Eq.(3.8) as:

$$
\begin{aligned}
& \frac{-p_{1}^{+}}{2 \pi} \int d x^{-} e^{-i x^{-} p_{1}^{+}}<H(P)\left|G^{b, \nu}(x)\left(\partial^{\alpha} \partial^{\beta} G^{b, \mu}(0)\right)\right| H(P)> \\
& =\left(d_{T}^{\mu \alpha} d_{T}^{\nu \beta}+d_{T}^{\mu \beta} d_{T}^{\nu \alpha}-d_{T}^{\mu \nu} d_{T}^{\alpha \beta}\right) \cdot d_{1}(z) \\
& \quad+\left(3 d_{T}^{\mu \nu} d_{T}^{\alpha \beta}-\left(d_{T}^{\mu \alpha} d_{T}^{\nu \beta}+d_{T}^{\mu \beta} d_{T}^{\nu \alpha}\right)\right) \cdot d_{2}(z) \\
& \quad+" H i g h \text { Twist Terms" }
\end{aligned}
$$


where the functions $d_{1}(z)$ and $d_{2}(z)$ are defined as

$$
\begin{aligned}
& d_{1}(z)=\frac{-1}{2 \pi z P^{+}} \int d x^{-} e^{-i z P^{+} x^{-}} \frac{1}{4}<H(P)\left|G_{\sigma}^{a,+}\left(x^{-} n\right)\left(D_{T}^{\{\sigma} D_{T}^{\rho\}} G_{\rho}^{+}(0)\right)^{a}\right| H(P)>, \\
& d_{2}(z)=\frac{-1}{2 \pi z P^{+}} \int d x^{-} e^{-i z P^{+} x^{-}} \frac{1}{8}<H(P)\left|G_{\sigma}^{a,+}\left(x^{-} n\right)\left(D_{T}^{2} G_{\sigma}^{+}(0)\right)^{a}\right| H(P)>.
\end{aligned}
$$

In Eq.(3.9) we replaced derivatives with covariant derivatives and $\partial_{T}^{\sigma} \partial_{T}^{\rho}$ with $D_{T}^{\{\sigma} D_{T}^{\rho\}}$. The symbol $\{\sigma \rho\}$ means to take the part symmetric in $\sigma \rho$. Calculating moments of the distributions $d_{1}(z)$ and $d_{2}(z)$ one can show that the moments correspond the matrix elements of local operators, which are the trace-terms subtracted in the twist-2 gluonic operators. We denote the twis- 4 contribution from Eq.(3.8) to the cross-section as $\sigma_{A 2}$, which is:

$$
\begin{aligned}
2 s d \sigma_{A 2} & =\frac{d^{4} k}{(2 \pi)^{4}}(2 \pi) \delta\left(k^{2}-M_{\psi}^{2}\right) \int \frac{d z}{z} \\
& \cdot\left\{d_{1}(z) \cdot\left[\left.\frac{1}{16} \cdot \frac{\partial^{2} S_{\mu \nu}\left(p_{1}\right)}{\partial p_{1}^{\alpha} \partial p_{1}^{\beta}}\right|_{p_{1}=\hat{p}_{1}}\right] \cdot\left(d_{T}^{\mu \alpha} d_{T}^{\nu \beta}+d_{T}^{\mu \beta} d_{T}^{\nu \alpha}-d_{T}^{\mu \nu} d_{T}^{\alpha \beta}\right)\right. \\
& \left.+d_{2}(z) \cdot\left[\left.\frac{1}{16} \cdot \frac{\partial^{2} S_{\mu \nu}\left(p_{1}\right)}{\partial p_{1}^{\alpha} \partial p_{1}^{\beta}}\right|_{p_{1}=\hat{p}_{1}}\right] \cdot\left(3 d_{T}^{\mu \nu} d_{T}^{\alpha \beta}-\left(d_{T}^{\mu \alpha} d_{T}^{\nu \beta}+d_{T}^{\mu \beta} d_{T}^{\nu \alpha}\right)\right)\right\}
\end{aligned}
$$

Some care must be taken for calculating this part. In $S_{\mu \nu}\left(p_{1}\right)$ there is a $\delta$-function $\delta\left(\left(q+p_{1}-k\right)^{2}\right)$, which is the on-shell condition for the gluon crossing the cut in Fig.2A. Therefore the derivatives of $S_{\mu \nu}\left(p_{1}\right)$ are constrained derivatives. To calculate the derivatives we first perform the integration of $z$ with the $\delta$-function in Eq.(3.11), where $\mathbf{p}_{\mathbf{1}} \mathbf{T}$ is not zero. After the integration $p_{1}^{+}$is fixed and it depends on $\mathbf{p}_{\mathbf{1} \mathbf{T}}$. Now the derivatives can be easily performed, where the indices $\alpha$ and $\beta$ are transversal indices. Finally we rewrite the result as an integration of $z$ with the $\delta$-function and set $p_{1}=\hat{p}_{1}$. The calculation is tedious but straightforward. The results for the perturbative part at order of $\alpha \alpha_{s}^{2}$ are:

$$
\begin{gathered}
\frac{d \sigma_{A 2}}{d t}=\frac{32 \pi}{3} \alpha \alpha_{s}^{2} Q_{Q}^{2} M_{\psi}\left|R_{\psi}(0)\right|^{2} \int \frac{d z}{z} \cdot \frac{1}{\hat{s}^{2}\left(\hat{s}-M_{\psi}^{2}\right)^{4}\left(\hat{t}-M_{\psi}^{2}\right)^{4}(\hat{s}+\hat{t})^{2}} \\
\cdot\left\{f_{1}(\hat{s}, \hat{t}) \cdot \frac{d_{1}(z)}{M_{\psi}^{2}}+f_{2}(\hat{s}, \hat{t}) \cdot \frac{d_{2}(z)}{\hat{s}}\right\}
\end{gathered}
$$


where the functions $f_{1}$ and $f_{2}$ are:

$$
\begin{aligned}
f_{1}(\hat{s}, \hat{t})= & 8 \hat{s}^{4} \hat{t}^{2}(\hat{s}+\hat{t})^{2}+16 M_{\psi}^{2} \hat{s} \hat{t}\left(-\hat{s}^{5}-5 \hat{s}^{4} \hat{t}-4 \hat{s}^{3} \hat{t}^{2}+2 \hat{s}^{2} \hat{t}^{3}+6 \hat{s} \hat{t}^{4}+4 \hat{t}^{5}\right) \\
& +8 M_{\psi}^{4}\left(\hat{s}^{6}+10 \hat{s}^{5} \hat{t}+21 \hat{s}^{4} \hat{t}^{2}-8 \hat{s}^{3} \hat{t}^{3}-48 \hat{s}^{2} \hat{t}^{4}-40 \hat{t}^{5}-8 \hat{t}^{6}\right)+16 M_{\psi}^{6}\left(-\hat{s}^{5}\right. \\
& \left.-9 \hat{s}^{4} \hat{t}+\hat{s}^{3} \hat{t}^{2}+35 \hat{s}^{2} \hat{t}^{3}+40 \hat{s} \hat{t}^{4}+14 \hat{t}^{5}\right)+8 M_{\psi}^{8}\left(-2 \hat{s}^{4}+8 \hat{s}^{3} \hat{t}-37 \hat{s}^{2} \hat{t}^{2}-78 \hat{s} \hat{t}^{3}\right. \\
& \left.-43 \hat{t}^{4}\right)+16 M_{\psi}^{10}\left(3 \hat{s}^{3}+14 \hat{s} \hat{t}^{2}+17 \hat{t}^{3}\right)+8 M_{\psi}^{12}\left(-3 \hat{s}^{2}+2 \hat{s} \hat{t}-11 \hat{t}^{2}\right), \\
f_{2}(\hat{s}, \hat{t})= & +32 \hat{s}^{2} \hat{t}\left(-2 \hat{s}^{5}-\hat{s}^{4} \hat{t}+2 \hat{s}^{3} \hat{t}^{2}+2 \hat{s}^{2} \hat{t}^{3}-2 \hat{s} \hat{t}^{4}-3 \hat{t}^{5}\right)+16 M_{\psi}^{2} \hat{s}\left(-2 \hat{s}^{6}+6 \hat{s}^{5} \hat{t}\right. \\
& \left.-3 \hat{s}^{4} \hat{t}^{2}-6 \hat{s}^{3} \hat{t}^{3}+5 \hat{s}^{2} \hat{t}^{4}+16 \hat{s} \hat{t}^{5}+2 \hat{t}^{6}\right)+32 M_{\psi}^{4}\left(4 \hat{s}^{6}+\hat{s}^{5} \hat{t}+2 \hat{s}^{4} \hat{t}^{2}-\hat{s}^{3} \hat{t}^{3}\right. \\
& \left.-8 \hat{s}^{2} \hat{t}^{4}-3 \hat{s} \hat{t}^{5}-\hat{t}^{6}\right)+32 M_{\psi}^{6}\left(-6 \hat{s}^{5}-2 \hat{s}^{4} \hat{t}+\hat{s}^{3} \hat{t}^{2}+3 \hat{s}^{2} \hat{t}^{3}+2 \hat{s} \hat{t}^{4}+3 \hat{t}^{5}\right) \\
& +32 M_{\psi}^{8}\left(4 \hat{s}^{4}-\hat{s}^{3} \hat{t}-\hat{s}^{2} \hat{t}^{2}+\hat{s} \hat{t}^{3}-3 \hat{t}^{4}\right)+32 M_{\psi}^{10}\left(-\hat{s}^{3}+\hat{s}^{2} \hat{t}-\hat{s} \hat{t}^{2}+\hat{t}^{3}\right) .
\end{aligned}
$$

At next-to-leading twist the contribution to the total cross-section for the process in Eq.(2.1) is the sum:

$$
\sigma^{(2)}=\sigma_{A 1}+\sigma_{A 2}
$$

In our results the nonperturbative parts at order of twist-4 in the process are the correlation functions $e_{1}(z), d_{1}(z)$ and $d_{2}(z)$, while that at order of leading twist is $f_{G / H}(z)$. These functions are universal, i.e., they will appear in other processes at certain order of $\alpha_{s}$, provided the same hadron $H$ is in the initial state. The functions $e_{1}(z), d_{1}(z)$ and $d_{2}(z)$ have a dimension 2 in mass and they are at order of $\Lambda_{Q C D}^{2}$. The perturbative parts corresponding to these functions are calculated at leading order of $\alpha$ and $\alpha_{s}$ in this section. Comparing the results with that in the last section we can see that the effect from next-to-leading twist is suppressed by the inverse of one scale which can be $\hat{s}, \hat{t}$ or $M_{\psi}^{2}$, or any linear combination of them. To see more clearly how the twist-4 effect is suppressed we consider two limiting cases, these cases can be realized with suitable cuts in experiment. One case is the kinematical region where $\hat{s},|\hat{t}|>>M_{\psi}^{2}$, but $\hat{t}$ is not so large that the $\psi$ does not appear in the forward region, whose definition will be given later. Another is for $\hat{s}>>|\hat{t}|, M_{\psi}^{2}$.

Case 1). $\hat{s},|\hat{t}|>>M_{\psi}^{2}$. In this case, one can neglect any power of $\frac{M_{\psi}^{2}}{\hat{s}}$ and $\frac{M_{\psi}^{2}}{\hat{t}}$. In this approximation the expression for the differential cross-section simplifies and the contribution related to $d_{2}(z)$ can be neglected:

$$
\begin{aligned}
\frac{d \sigma}{d t}= & \frac{32 \pi}{3} \alpha \alpha_{s}^{2} Q_{Q}^{2} M_{\psi}\left|R_{\psi}(0)\right|^{2} \int \frac{d z}{z} \cdot \frac{1}{\hat{s}^{2} \hat{t}^{2}} \\
& \cdot\left\{\frac{\hat{s}^{4}+\hat{t}^{4}+(\hat{s}+\hat{t})^{4}}{\hat{s}^{2}(\hat{s}+\hat{t})^{2}} \cdot f_{G / H}(z)+2 \frac{e_{1}(z)}{M_{\psi}^{2}}+8 \frac{d_{1}(z)}{M_{\psi}^{2}}\right\} .
\end{aligned}
$$


From Eq.(3.15) we clearly see that the twist-4 effect is suppressed only by $M_{\psi}^{-2}$, but not by the inverse of any large scale of $\hat{s}$ or $\hat{t}$.

Case 2). $\hat{s}>>M_{\psi}^{2},|\hat{t}|$. In this limit we neglect any power of $\frac{M_{\psi}^{2}}{\hat{s}}$ and $\frac{\hat{t}}{\hat{s}}$. The differential cross-section becomes

$$
\begin{aligned}
\frac{d \sigma}{d t}= & \frac{32 \pi}{3} \alpha \alpha_{s}^{2} Q_{Q}^{2} M_{\psi}\left|R_{\psi}(0)\right|^{2} \int \frac{d z}{z} \cdot \frac{1}{\hat{s}^{2}\left(\hat{t}-M_{\psi}^{2}\right)^{2}} \\
& \cdot\left\{2 f_{G / H}(z)+2 \frac{e_{1}(z)}{M_{\psi}^{2}}+8 \frac{d_{1}(z)}{M_{\psi}^{2}}-32 \frac{\left(M_{\psi}^{2}+2 \hat{t}\right)}{\left(\hat{t}-M_{\psi}^{2}\right)^{2}} \cdot d_{2}(z)\right\}
\end{aligned}
$$

Again the twist- 4 effect here is suppressed by $M_{\psi}^{-2}$ or by the inverse of a linear conbination of $M_{\psi}^{2}$ and $\hat{t}$.

In general there is always some higher twist effect which is suppressed only by $M_{\psi}^{-2}$. From the discussions of the above two cases one can not make this effect negligible through suitable cuts in experiment. Therefore the twist-4 effect may be substantial for charmonium production, as the mass $M_{c}$ is not so large. Because the nonperturbative functions $e_{1}(z)$, $d_{1}(z)$ and $d_{2}(z)$ are unknown, one does not know how large the twist- 4 effect can be. A naive estimation could be given by noting the fact that the effect we considered is basically from the correction of the parton-model, where one takes the off-shellness and the transversal momentum of the parton into account. With this fact one may make an Ansatz for a naive estimation as follows:

$$
e_{1}(z)=d_{1}(z)=d_{2}(z)=\Lambda^{2} f_{G / H}(z)
$$

where the parameter $\Lambda$ can be regarded as an average of the transversal momentum of the initial gluon and it should be at the order corresponding the radius of the initial hadron. With this Ansatz the differential cross-section upto twist-4 can be written:

$$
\frac{d \sigma}{d t}=\frac{32 \pi}{3} \alpha \alpha_{s}^{2} Q_{Q}^{2} M_{\psi}\left|R_{\psi}(0)\right|^{2} \int \frac{d z}{z}\left(w_{2}(\hat{s}, \hat{t})+\Lambda^{2} w_{4}(\hat{s}, \hat{t})\right) \cdot f_{G / H}(z)
$$

We will give some numerical results for the quantity $\rho$ defined as

$$
\rho=\frac{w_{2}(\hat{s}, \hat{t})+\Lambda^{2} w_{4}(\hat{s}, \hat{t})}{w_{2}(\hat{s}, \hat{t})}
$$

If the twist- 4 effect is negligible, $\rho$ should be close to 1 . An useful variable used commonly in experiment is $z$, it is defined as:

$$
z=\frac{k \cdot P}{q \cdot P}
$$


which should not be confused with the intergration variable $z$ in previous equations. If $\psi$ is produced with $z>0.9$, it is in the froward region. We plot the quantity $\rho$ as a function of $z$ for a given $\hat{s}$, where we take $\Lambda=500 \mathrm{MeV}$, this value may correspond to a proton as the initial hadron. The results are given in Fig. 3 for $\hat{s}=(10 \mathrm{GeV})^{2}$ and for $\hat{s}=(30 \mathrm{GeV})^{2}$ respectively and the quarkonium is $J / \psi$. On can take these as representative estimations for $\sqrt{s}=30 \mathrm{GeV}$ and $\sqrt{s}=100 \mathrm{GeV}$. From the figure we note that the twist- 4 effect becomes smaller when $\hat{s}$ becomes larger and it is more significant in lower $z$-region than that in higher $z$-region. For $\hat{s}=(30 \mathrm{GeV})^{2}$ it can be an effect at $30 \%$.

\section{Higher Order Effect in the Small Velocity Expansion}

In previous sections we analyzed the nonperturbative effect at twist- 4 related to the initial hadron, while the nonperturbative effect related to the quarkonium is only considered at leading order of the small velocity expansion. In this section we discuss higher order effect in this expansion. At leading order of $v$ only a color-singlet $Q \bar{Q}$ pair in a ${ }^{3} S_{1}$ state can be transmitted into the ${ }^{3} S_{1}$ quarkonium, the probability for this can be regarded as $\left|R_{\psi}(0)\right|^{2}$, which should be related to a matrix element defined in NRQCD[2]. The relation is

$$
\left|R_{\psi}(0)\right|^{2}=\frac{2 \pi}{9}<0\left|O_{1}^{\psi}\left({ }^{3} S_{1}\right)\right| 0>
$$

We will not give the definition of the matrix element and of those appearing later. For the definitions we refer reader to [2]. We will take the leading order here as $v^{0}$. Corrections to the leading order results will start at order of $v^{2}$.

At next-to-leading order, i.e., at $v^{2}$, there is only the relativistic correction, which takes the effect of the relative motion of $Q$ and $\bar{Q}$ into account. This effect is analyzed within the color-singlet model in [14], where the correction is parameterized in term of the parameter $\epsilon$ defined as:

$$
M_{\psi}=2 M_{Q}+\epsilon
$$

Numerical results given in [14] show that for charmonium the correction at $\sqrt{s}=14.7 \mathrm{GeV}$ is significant in the high $z$ region, where $z$ is defined in Eq.(3.20) and $\epsilon=0.16 M_{c}$. However, there is a uncertainty in choosing the parameter $\epsilon$. If one thinks that the binding force is Couloumb force, $\epsilon$ defined in Eq.(4.2) is negative. One can also show by starting the energymomentum tensor the relation that $\epsilon$ equals to the negative matrix element in NRQCD of the kinetic-energy operator of $Q$ or $\bar{Q}$ inside the quarkonium, if $M_{Q}$ in Eq.(4.2) is the polemass[15]. However, this relation holds at the tree-level and at the leading order of $v$. All 
these show that $\epsilon$ can also be negative. With a negative $\epsilon$ the correction turns into negative too. It should be pointed out that the analyze in [14] is done with color-singlet model. With the new NQRCD factorization in [2] the nonrelativistic correction is parameterized with other type of matrix elements instead of $\epsilon$ and a re-analyze seems needed.

At order of $v^{4}$, except the relativistic corrections there are also other corrections due to the fact that a quarkonium state consists many components, where a $Q \bar{Q}$ pair carrying the quantum numbers of the quarkonium is the dominant one. Other components consists fo a $Q \bar{Q}$ pair and soft glue, the probability to find them in a quarkonium is suppressed by powers of $v$ relatively to that of the dominant one. Because of this a produced $Q \bar{Q}$ with quantum numbers other than those of the quarkonium can become one of these components by combining soft glue and hence the production of such $Q \bar{Q}$ pair will also contribute to the production rate of the quarkonium. In our case such $Q \bar{Q}$ pair can be a color-octet $Q \bar{Q}$ pair in ${ }^{1} S_{0},{ }^{3} S_{1}$ or ${ }^{3} P_{J}$ state, where $J=0,1,2$. We use the notation $Q \bar{Q}_{(8)}\left({ }^{2 S+1} L_{J}\right)$ to denote these states. With the velocity power counting rule given in [16] and the method in [2] one can show that the contributions from these color-octet $Q \bar{Q}$ pairs are at order of $v^{4}$. Taking the state $Q \bar{Q}_{(8)}\left({ }^{3} P_{1}\right)$ as an example, the probability to find such state combined with a soft gluon as a component of the quarkonium is at order of $v^{2}$, while the formation of $Q$ and $\bar{Q}$ into a $P$-wave state has a chance at order of $v^{2}$, hence the production of the quarkonium through such state will happen at order of $v^{4}$.

We first discuss the contribution from a $Q \bar{Q}_{(8)}\left({ }^{3} S_{1}\right)$ state. Such state can be produced not only by photon-gluon fusion at order $\alpha \alpha_{s}^{2}$, but also by photon-quark scattering at the same order. However, numerical results show that the contribution from the photon-quark scattering is negligible. It is interesting to note that the contribution from the photon-gluon fusion is represented by the same diagrams for the color-singlet contribution worked out before. To include this contribution one needs only to replace the matrix element in our previous results via:

$$
<0\left|O_{1}^{\psi}\left({ }^{3} S_{1}\right)\right| 0>\rightarrow<0\left|O_{1}^{\psi}\left({ }^{3} S_{1}\right)\right| 0>+\frac{5}{6} \cdot<0\left|O_{8}^{\psi}\left({ }^{3} S_{1}\right)\right| 0>.
$$

where the NRQCD matrix element $<0\left|O_{8}^{\psi}\left({ }^{3} S_{1}\right)\right| 0>$ is for the transition of the color-octet $Q \bar{Q}$. Due to the uncertainties in matrix elements and others like effects in higher order of $\alpha_{s}, K$-factor problem $\cdots$ etc, it is hard to identify this color-octet contribution.

The contributions from the $Q \bar{Q}_{(8)}\left({ }^{1} S_{0}\right), Q \bar{Q}_{(8)}\left({ }^{3} P_{J}\right)$ state at leading twist are analyzed in $[17,18,19]$ for $J / \psi$. The matrix element for the transition of these states into $J / \Psi$, i.e., $<0\left|Q_{8}^{\psi}\left({ }^{1} S_{0}\right)\right| 0>$ and $<0\left|O_{8}^{\psi}\left({ }^{3} P_{J}\right)\right| 0>$, is estimated[19,20]. The situation here is controversial. In the forward region, the contributions are from the subprocess $\gamma+G \rightarrow Q+\bar{Q}$ 
at the order of $\alpha \alpha_{s}$. In experiment the measured cross section contains also contributions from elastic diffractive processes which can not be described with perturbative QCD. That means that the cross section estimated from the subprocess should be smaller than the measured one, if one neglects all higher order effects. A comparison with experiment for $J / \psi$ production shows that the predicted cross section from the subprocess[17] and also including color-singlet contributions[18] is much larger than measured one, if one uses the numerical values for matrix elements estimated from experiment at Tevatron[20], they are roughly:

$$
\begin{aligned}
& <0\left|Q_{8}^{\psi}\left({ }^{1} S_{0}\right)\right| 0>\sim 10^{-2} \mathrm{GeV}^{3} \\
& \frac{<0\left|O_{8}^{\psi}\left({ }^{3} P_{0}\right)\right| 0>}{M_{c}^{2}}=\frac{1}{2 J+1} \cdot \frac{<0\left|O_{8}^{\psi}\left({ }^{3} P_{J}\right)\right| 0>}{M_{c}^{2}} \sim 10^{-2} \mathrm{GeV}^{3} .
\end{aligned}
$$

It seems that these matrix elements are overestimated at least by one order of magnitude. Various reasons for that discrepancy are proposed like corrections from higher order of $\alpha_{s}$ and uncertainties in extracting numerical values of the matrix elements. One should also note that the predictions may suffer large uncertainties because of twist- 4 effect, as this effect is only suppressed by $M_{Q}^{-2}$ in the forward region. In addition, the relativistic correction at order of $v^{2}$ discussed before is significant in the froward region and can be negative.

In the nonforward region, these color-octet states can be produced at order of $\alpha \alpha_{s}^{2}$. At leading twist the production is through photon-gluon fusion described by Fig.1A, where the blank part represents not only the Feynman diagrams given in Fig.2A, but also those like the one given in Fig.4, in which the gluon in the final state is emitted by the incoming gluon. A comparison with available experiments is made in this region in $[17,18]$. It is shown that the contribution from these color-octet states becomes dominant for $z>0.5$ and including this contribution leads to that the differential cross section increases rapidly as $z$ increases. This rapid increasing however is not favoured by experimental data[17,18]. If the relativistic correction discussed before is negative, the increasing may become mild. An important question related to our work is whether there is some twist-4 effect suppressed only by $M_{Q}^{-2}$ as in the color-singlet contribution or not. The answer is no. To see this one needs to understand why the twist-4 effect analyzed before is suppressed by $M_{Q}^{-2}$. If one expands the perturbative part at leading twist in $M_{Q}$, the leading power is 2 . In the final result in Eq.(2.14) an extra $M_{Q}^{-1}$ comes in because of the normalization. The leading power of the perturbative parts at twist- 4 is 0 . Therefore there is twist- 4 effect suppressed only by $M_{Q}^{-2}$ in the production of a ${ }^{3} S_{1}$ quarkonium. For the above color-octet states the leading power of the perturbative parts at leading twist is zero or negative, and in calculating perturbative parts at twist-4 extra negative power in $M_{Q}$ can not be generated, so there 
will be no twist- 4 effect which is suppressed only by $M_{Q}^{-2}$ like those in Eq.(3.15).

Before ending this section we briefly discuss electroproduction. This is basically the process $\gamma^{*}+H \rightarrow \psi+X$ where the photon $\gamma^{*}$ is off-shell and the off-shellness is $-Q^{2}$. One can perform an analysis of twist-4 effect as we did here for a real photon. One can show for a fixed $Q^{2}$ that there is no twits-4 effect suppressed by $M_{Q}^{-2}$. The reason is the same as for the color-octet states mentioned above. In photoproduction the forward region is the kinematical region where the produced quarkonium moves roughly in the same direction as the photon beam in a fixed target experiment. In this region the contribution from elastic and diffractive processes to the production rate is significant. In electroproduction like HERA experiment the produced quarkonium in the forward region can have large angle in respect to the hadron beam, one may expect that the contribution from elastic and diffractive processes may not be significant. If the events in this region can be described by perturbative QCD, the theoretical predictions indicate that there is a peak in the forward region, resulted by color-octet states produced by the subprocess $\gamma^{*}+G \rightarrow Q+\bar{Q}$ and the subprocess at twist- 4 described by Fig. $1 \mathrm{C}$. In experiment $\mathrm{NMC}$ data of $J / \psi$-production shows that there really is a peak in the forward region[21]. However, the data was given as the rapidity distribution, hence many information was lost for a detail study of the peak. On the other hand, it is also pointed out in the second reference in [11] that the peak can be well explained by the theoretical results at leading twist. If more data in experiment is available, this region may be a good place to study the effect from those color-octet states and from twist-4.

\section{Summary}

In this work we have analyzed twist- 4 effect in photoproduction of a ${ }^{3} S_{1}$ quarkonium. The nonperturbative parts at order of twist- 4 are factorized into 3 correlation functions related to the initial hadron. The perturbative parts are calculated at leading order of coupling constants. We find that the twist-4 effect suppressed by $M_{Q}^{-2}$ in comparison with that at leading twist. Hence the twist- 4 effect in photoproduction of $J / \psi$ may be substantial, and it can not be made negligible by experimental cuts. Naive estimations indicate that the effect is significant at $\sqrt{s}$ around several ten GeVs. However, a definite conclusion requires a detailed study.

In the analysis we only take the leading order of the small velocity for the nonperturbative effect related to the quarkonium. The effects from higher orders of $v$ upto $v^{4}$ are discussed in detail. Especially, at order of $v^{4}$ production of various color-octet states of $Q \bar{Q}$ can contribute to the total production rate. However, the contributions from these states 
will not receive from twist-4 corrections suppressed only by $M_{Q}^{-2}$ in the nonforward region, except the contribution from the color-octet state $Q \bar{Q}_{(8)}\left({ }^{3} S_{1}\right)$. But it is hard to distinguish this contribution from those of the color-singlet as discussed in the last section. We have also discussed some aspects in electroproduction, here the twist-4 corrections are also not suppressed by $M_{Q}^{-2}$. It seems that the appearance of such suppression in photoproduction of ${ }^{3} S_{1}$ quarkonium may be a special case.

Unlike the gluon distribution $f_{G / H}(z)$, which is measured in experiment in many places, the three correlation functions for the nonperturbative effect of twist-4 are unknown and need to be measured. These three functions are defined in Sect.3 in the light-cone gauge. In arbitrary gauge one needs to supply Wilson-line operators between the gluonic operators in these definition. It should be noted that the definitions in Sect.3 are unrenormalized versions, i.e., at tree level. A definition in renormalized version like that for correlation functions at twist-2 given in [13] may be difficult. The main obstacles are that these functions may have power divergences and the perturbative parts corresponding to correlation functions at twist2 may have problem with convergence due to possible presence of renormalon. The later indicates that there may be still some nonperturbative effect contained in the perturbative part at leading twist. A perfect definition would be given so that the two problems would be solved(See discussions in [22]).

Finally, it should be noted that the twist-4 effect in the kinematic region considered here is basically from the corrections of the parton model, where one takes the transverse momentum and the off-shellness of incoming partons into account. With this in mind it is possible to make a model for a more realistic estimation of twist-4 effect than the naive estimations given in Sect.3. Currently a model is under development in hope to study the effect in detail. 


\section{References}

[1] E. Braaten, S. Fleming and T.C. Yuan, OHSTPY-HEP-T-96-001, hep-ph/96-02374 M. Beneke, Talk given in the Second Workshop on Continous Advances in QCD, hep-ph/9605462

[2] G.T. Bodwin, E. Braaten and G.P. Lepage, Phys. Rev. D51 (1995) 1125

[3] R.K. Ellis, W. Furmanski and P. Petronzio, Nucl. Phys. B212 (1983) 29

[4] R.L. Jaffe and M. Soldate, Phys. Lett. B105 (1981) 467, Phys. Rev. D26 (1982) 49

H.D. Politzer, Nucl. Phys. B172 (1980) 349

[5] J. Qiu, Phys. Rev. D42 (1990) 30

[6] R.L. Jaffe and X. Ji, Nucl. Phys. B375 (1992) 527

X.Ji, Nucl. Phys. B402 (1993) 217, Phys. Rev. D49 (1994) 114

[7] J. Qiu and G. Sterman, Nucl. Phys. B353 (1991) 105, ibid 137

[8] M. Luo, J. Qiu and G. Sterman, Phys. Lett. B279 (1992) 377,

Phys. Rev. D49 (1994) 4493 , ibid D50 (1994) 1951

[9] J. Levelt and P.J. Mulders, Phys. Rev. D49 (1994) 96

[10] J.P. Ma, Nucl. Phys. B460 (1996) 109

H. Khan and P. Hoodbhoy, Phys. Rev. D53 (1996) 2534

[11] A.D. Martin, C.-K. Ng and W.J. Stirling, Phys. Lett. B191 (1987) 200

H. Merabet, J.-F. Mathiot and R. Mendez-Galain, Z. Phys. C62 (1994) 639

[12] O. Nachtmann, Nucl. Phys. B63 (1973) 237

[13] J.C. Collins and D.E. Soper, Nucl. Phys. B194 (1982) 445

[14] H. Jung et al. Z. Phys. C60, (1993) 721

[15] J.P. Ma and B.H.J. McKellar, Preprint UM-P-96/55

[16] G.P. Lepage et al., Phys. Rev. D46 (1992) 4052

[17] P. Ko, J. Lee and H.S. Song, Phys. Rev. D54 (1996) 4312

[18] M. Cacciari and M. Krämer, Phys. Rev. Lett. 76 (1996) 4128

[19] J. Amundson, S. Fleming and I. Maksymyk, Preprint MADTH-95-914, hep-ph/9601289

[20] P. Cho and A.K. Leibovich, Phys. Rev. D53 (1996) 6203

[21] D. Allasia et al.; Phys. Lett. B258 (1991) 493

Ch. Mariotti, Nucl. Phys. A532 (1991) 437

[22] A.H. Mueller, Phys. Lett. B308 (1993) 355

X. Ji, Nucl. Phys. B448 (1995) 51 


\section{Figure Caption}

Fig.1A: The diagram appearing in the diagram expansion. This diagram leads to contributions at leading twist and at higher twist. The wavy lines are for gluons. The lines connecting the black box with the upper part denote only momentum flows and contractions of color- and Lorentz-indices.

Fig.1B: One of the two diagrams leads to contributions at next-to-leading twist. In these diagrams the upper part is connected to the black box with three gluon lines.

Fig.1C: The diagram leads to contributions at next-to-leading twist. the momentum flows are given with $k_{4}=k_{1}+k_{2}-k_{3}$.

Fig.2A: A diagram corresponds to the upper part in Fig.1A. Other diagrams are obtained through permutations of gluon- and photon-lines. The line is for heavy quark. The black dot denotes the nonperturbative transition into a quarkonium. The thick line is for quarkonium. Fig.2B: A diagram corresponds to the upper part in Fig.1B. Other diagrams are obtained through permutations of gluon- and photon-lines.

Fig.2C: A diagram corresponds to the upper part in Fig.1C. Other diagrams are obtained through permutations of gluon- and photon-lines.

Fig.3: The quantity $\rho$ as function of $z$ defined in Eq.(3.20). The line is for $\hat{s}=(10)^{2} \mathrm{GeV}$, the dashed one is for $\hat{s}=(30)^{2} \mathrm{GeV}$.

Fig.4: One of possible diagrams in addition to those in Fig.2A contributes to the production rate through color-octet states 


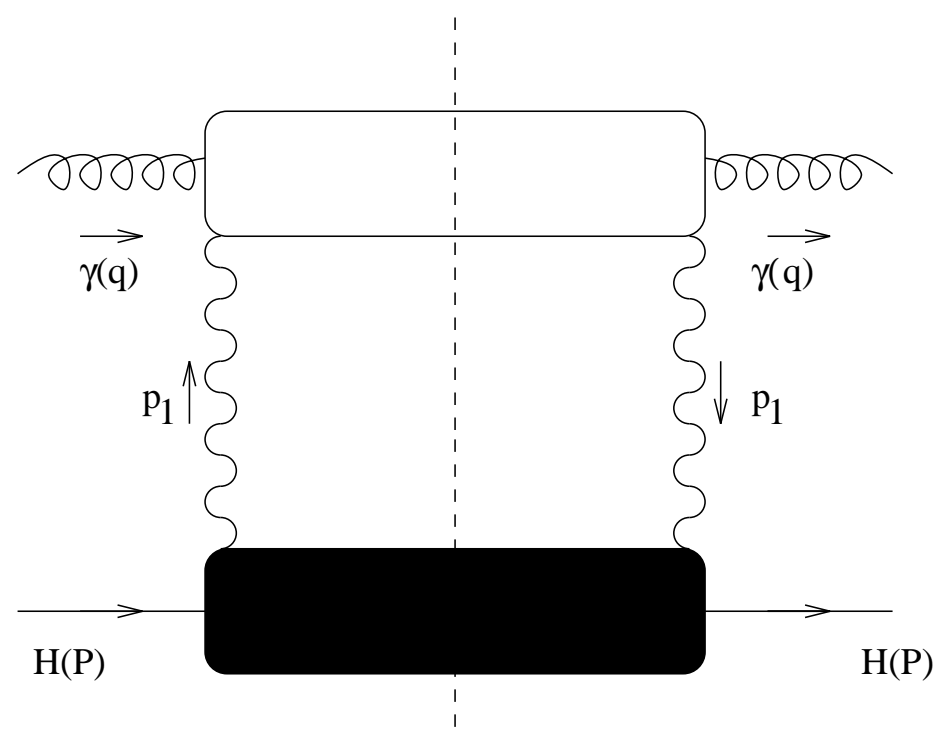

Fig.1A 


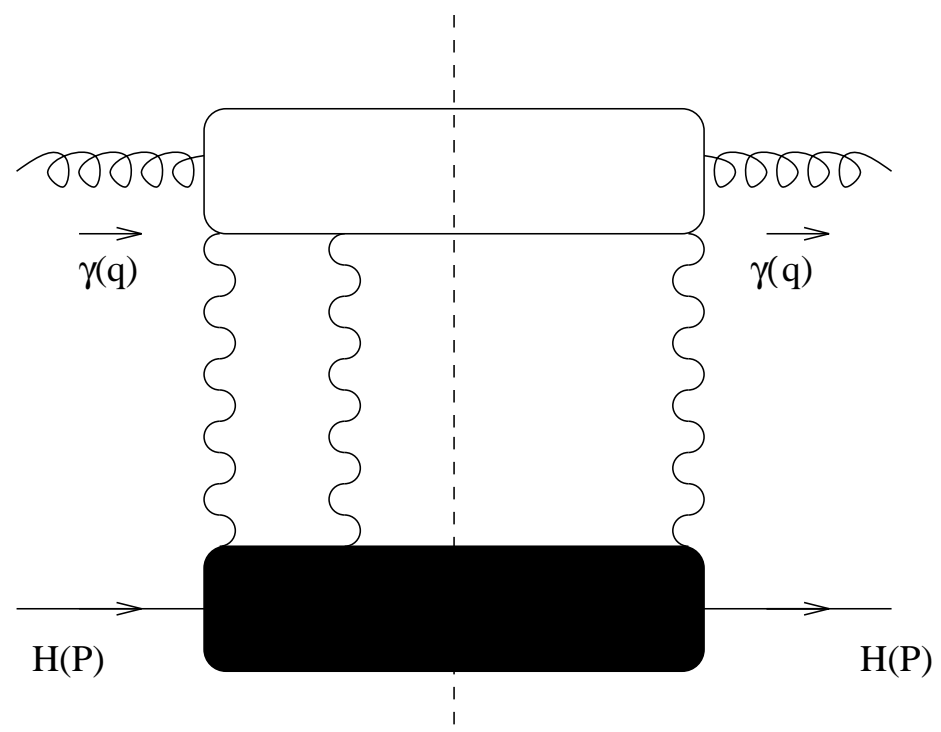

Fig.1B 


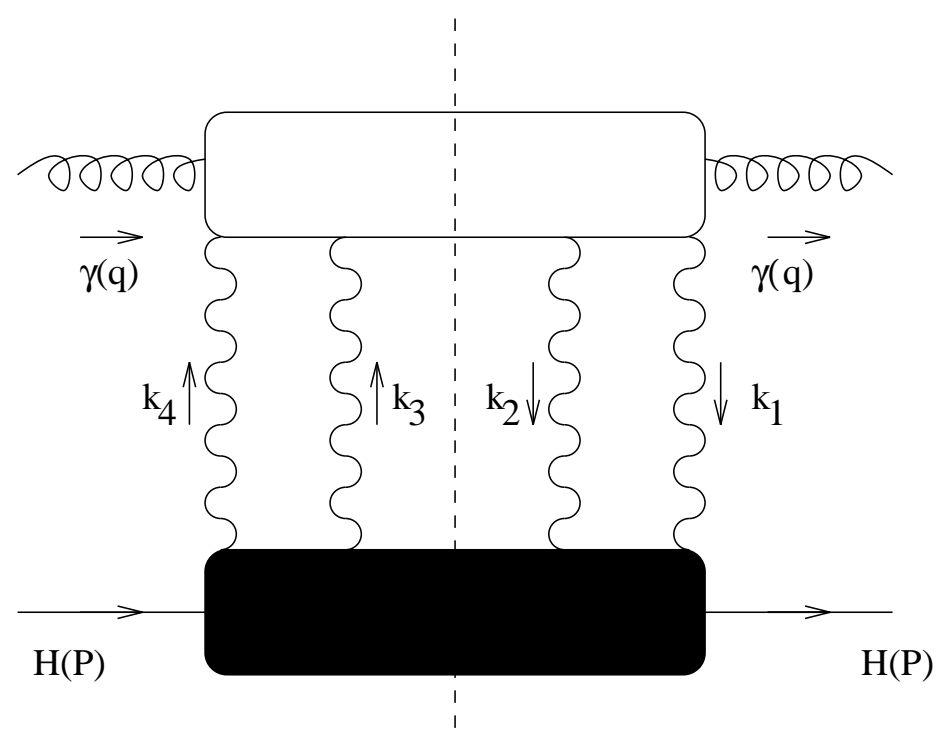

Fig.1C 


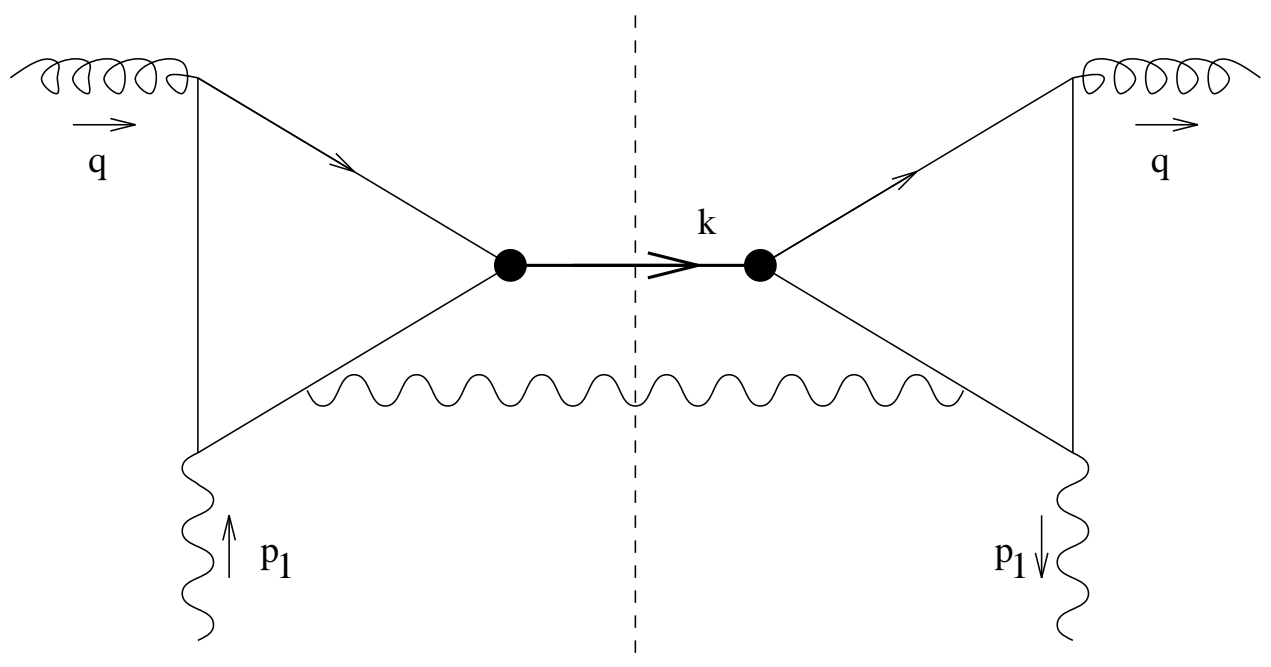

Fig.2A 


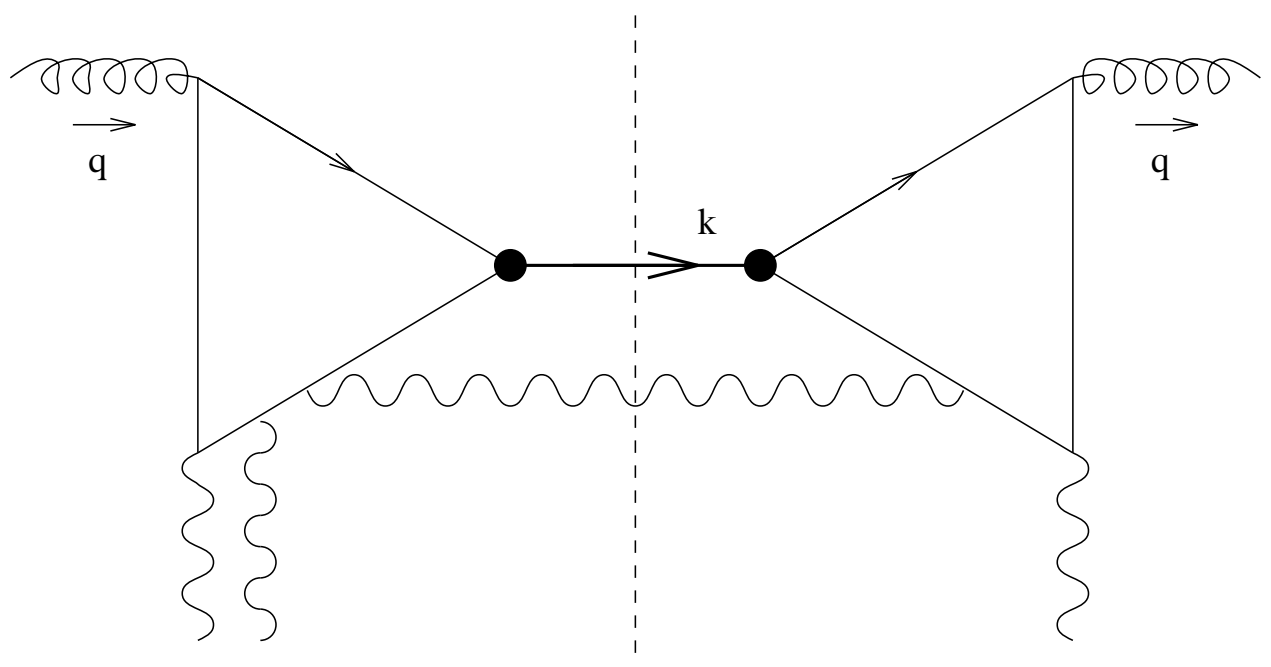

Fig.2B 


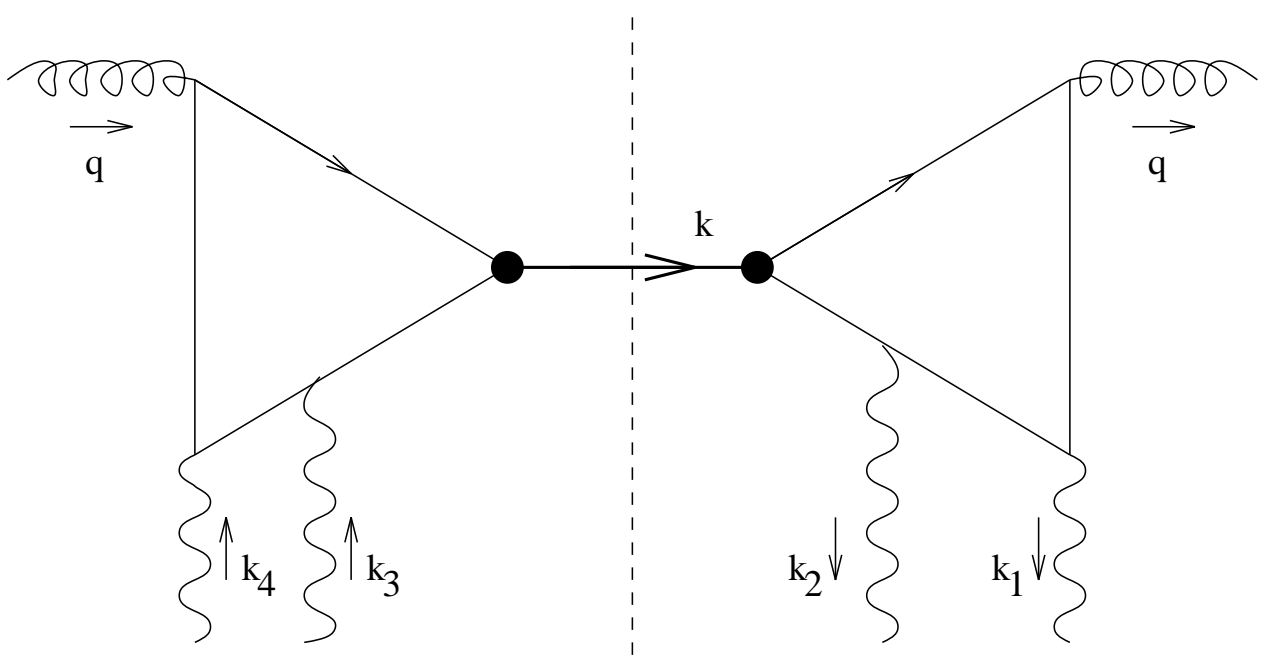

Fig.2C 


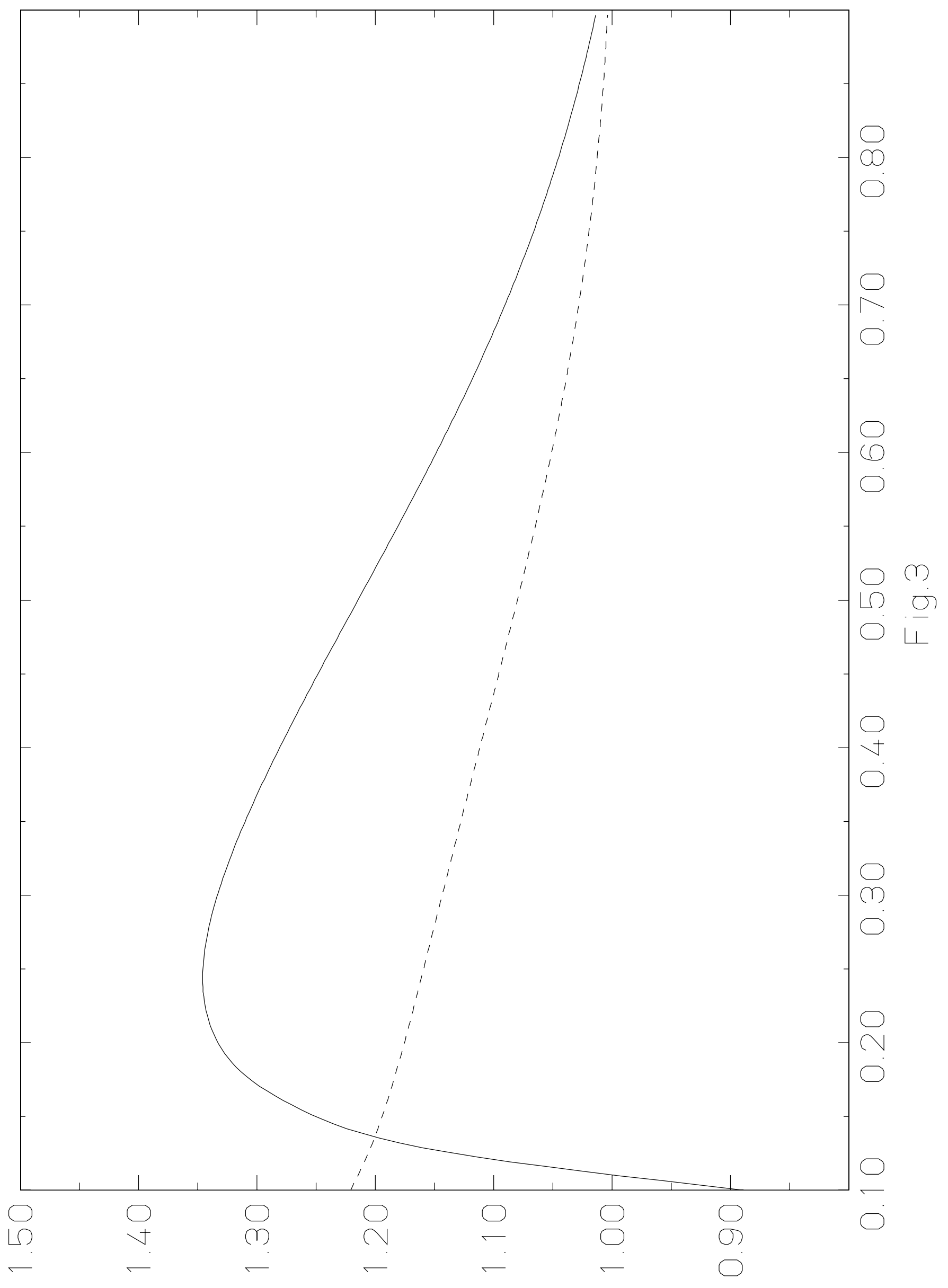




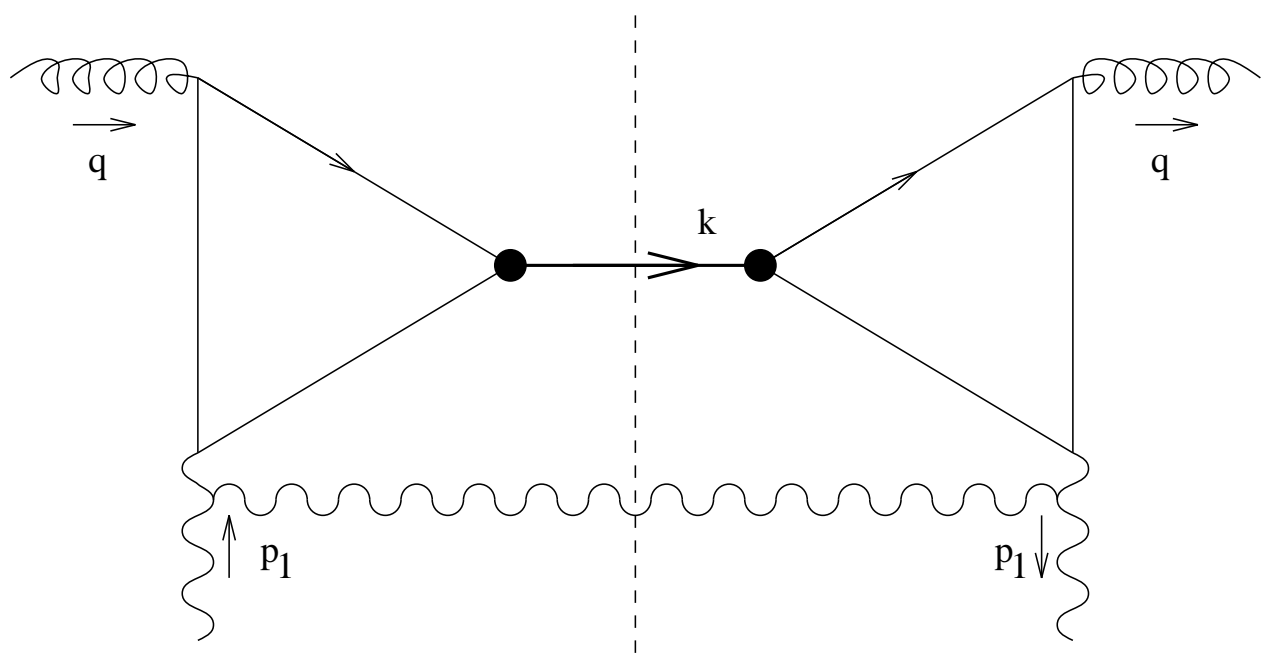

Fig.4 